\title{
DNA barcoding reveals commercial fraud related to yak jerky sold in China
}

\author{
Yunyu Wang ${ }^{1 \dagger}$, Kai He ${ }^{1 \dagger}$, Long Fan ${ }^{2 \dagger}$, Yushui Wang ${ }^{1,3}$, Shifang $\mathrm{Wu}^{1}$, Robert W Murphy ${ }^{1,4}$, \\ Wenzhi Wang ${ }^{1,3,5^{*}}$ \& Yaping Zhang ${ }^{1,6^{*}}$ \\ ${ }^{1}$ State Key Laboratory of Genetic Resources and Evolution and Yunnan Laboratory of Molecular Biology of Domestic Animals, Kunming In- \\ stitute of Zoology, Chinese Academy of Sciences, Kunming 650223, China; \\ ${ }^{2}$ Key Laboratory of Animal Models and Human Disease Mechanisms, Kunming Institute of Zoology, Chinese Academy of Sciences, \\ Kunming 650223, China; \\ ${ }^{3}$ Forensic Science Service of Yunnan Endangered Species Scientific Commission, Kunming 650223, China; \\ ${ }^{4}$ Centre for Biodiversity and Conservation Biology, Royal Ontario Museum, Toronto M5S 2C6, Canada; \\ ${ }^{5}$ Guizhou Academy of Testing and Analysis, Guiyang 550002, China; \\ ${ }^{6}$ Laboratory for Conservation and Utilization of Bio-resource and Key Laboratory for Microbial Resources of the Ministry of Education, \\ Yunnan University, Kunming 650091, China
}

Received September 9, 2015; accepted November 9, 2015

Citation: Wang, Y., He, K., Fan, L., Wang, Y., Wu, S., Murphy, R.W., Wang, W., and Zhang, Y. (2016). DNA barcoding reveals commercial fraud related to yak jerky sold in China. Sci China Life Sci 59, 106-108. doi: 10.1007/s11427-015-4979-0

Dear Editor,

Instances of food mislabeling and adulteration scandals focus public attention on food safety and market fraud. Such scandals force the authorities to enforce stringent regulations on food adulteration. The Chinese government and consumers are paying more attention to food resource quality and safety regulation than ever before. The growing food market requires food authenticity at all stages of product processing, while species identification of raw materials is fundamental for food safety. Species substitution causes economical loss to consumers and may negatively affect public sanitation because of possible presence of toxic and parasite-infected species (Galimberti et al., 2013). Globally, there is still inadequate regulation for species identification in food products, such as sliced and minced jerky. The main problem is that, after food processing, livestock products are morphologically indistinguishable because of the lack of discriminative features such as skull, fur, and tails. The recently developed technique of DNA barcoding has been

$\dagger$ Contributed equally to this work

*Corresponding authors (email: wangwz@mail.kiz.ac.cn; zhangyp@mail.kiz.ac.cn) proposed as a reliable, effective, and rapid technology for species identification (Hebert et al., 2003). DNA barcoding uses a short, standardized region to distinguish different taxa at the species level. Since DNA barcoding enables species identification for specimens lacking morphological features, it could be a convenient tool for tracing the raw materials of food at different stages of food industrial processing (Wallace et al., 2012).

Yaks (Bos grunniens) live throughout the Tibetan plateau and have been domesticated for thousands of years (Qiu et al., 2012). Because of its the small size of the domesticated population of yaks and the high quality of their meat, the price of yak meat is 2-10 times higher than that of cattle and buffalo meat (Genearal Administration of Quality Supervision, 2010). The possibility of market substitution in yak meat products in China and the effectiveness of DNA barcoding in identifying such frauds need to be investigated.

To address these issues, 30 different types/brands of commercial yak jerky products were purchased from commercial markets in four cities of China: Linzhi, Tibet $(n=2)$; Chengdu, Sichuan $(n=8)$; Kunming, Yunnan $(n=11)$; and Xining, Qinghai ( $n=9$ ) (Figure 1). In addition, three different types/brands of commercial beef jerky products were 


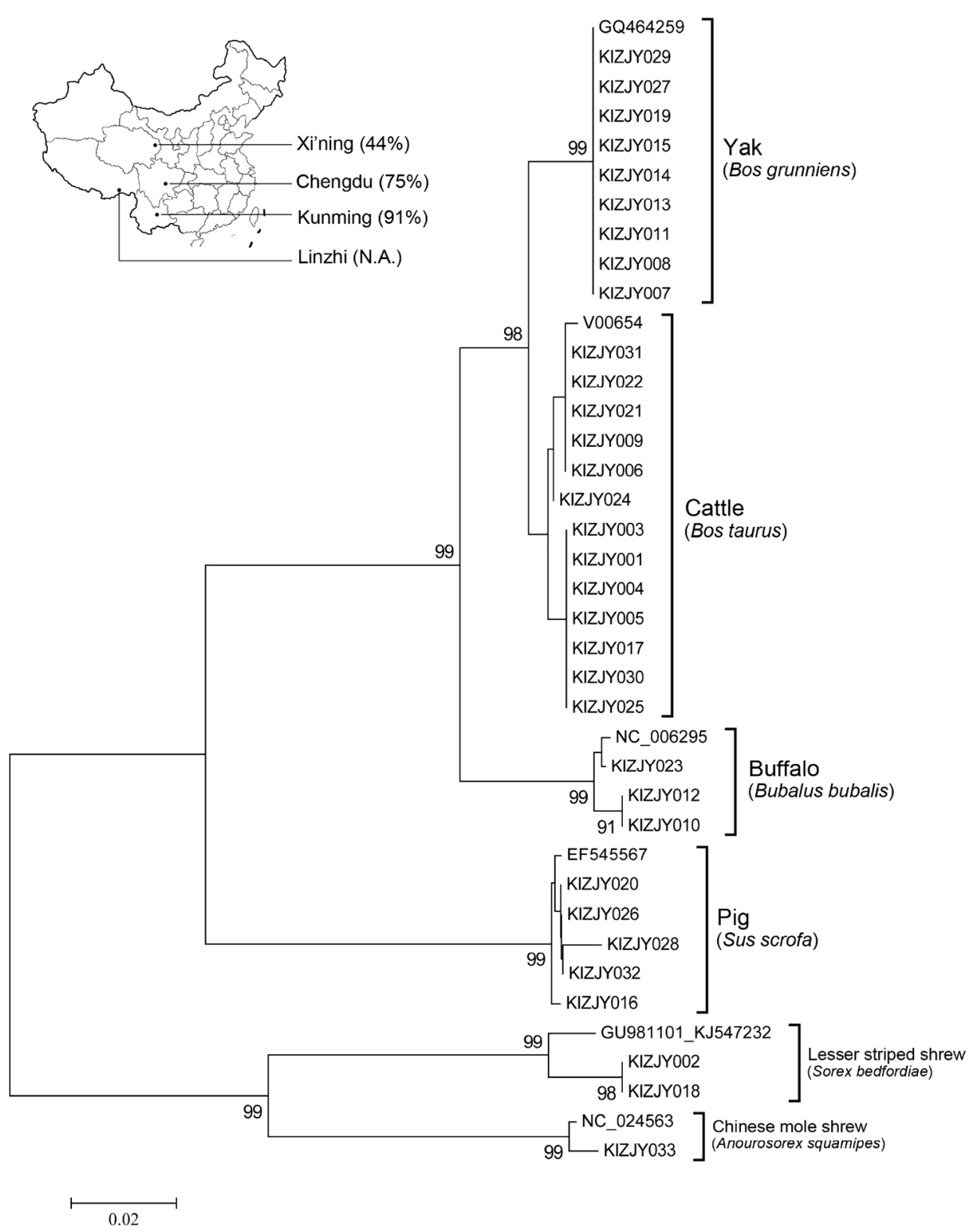

Figure 1 Sampling map and the neighbor-joining tree. The left corner shows the sampling map and the estimated fraud ratios for each site (N.A.: sample size too small for estimation). The main part of the figure shows the neighbor-joining tree of combined 16S and COI.

purchased in Kunming for use as controls. Details for all samples have been summarized in Table S1. The genes encoding cytochrome $c$ oxidase subunit 1 (COI) (Wallace et al., 2012) and 16S ribosomal RNA (16S rRNA) (Chen et al., 2010) were selected as barcoding markers to detect the substitution and adulteration in samples. All $16 \mathrm{~S}$ amplicons and $27 \mathrm{COI}$ amplicons obtained for the 33 samples were successfully sequenced, while the reaction for COI-5P was not successful for the other six samples due to poor DNA quality (see methods online for details). The images of the specimen, raw trace files, and sequences were uploaded on the Barcode of Life System (BOLD, www.boldsystems.org) (Ratnasingham and Hebert, 2007) with the project code "YAKJC". Sequences were also uploaded on GenBank with the accession numbers KT827187-KT827246.

All 16S and 27 COI-5P sequences of the 30 yak jerky samples and the three beef jerky samples were successfully determined. The success rates reached $100 \%, 80 \%$, and $100 \%$ for $16 \mathrm{~S}, \mathrm{COI}$, and concatenation of $16 \mathrm{~S}$ and COI, respectively. Using concatenation of $16 \mathrm{~S}$ and $\mathrm{COI}$ sequences, we successfully identified all 33 samples to the species level. Both BLAST (Table S1) and the neighbor-joining tree (NJ tree, Figure 1)-based method provided identical results. Only nine yak jerky samples were identified as yak (Bos grunniens) samples, whereas the other 21 were found to be adulterated (70\%). Among the substituted samples, 18 (60\%) were identified as beef meat (Bos taurus), buffalo beef (Bubalus bubalis), or pig meat (Sus scrofa). The other 
three samples (10\%) were identified as those of small mammals, including the lesser striped shrew (Sorex bedfordiae) and the Chinese mole shrew (Anourosorex squamipes). All three samples of beef jerky were prepared from beef. Briefly, $90 \%, 75 \%$, and $56 \%$ of yak jerky products were fraudulent in consumer markets of Kunming, Cheng$\mathrm{du}$, and Xining, respectively.

To summarize, we present a study using the DNA barcoding approach to identify species in samples of yak jerky, a thermally processed food. The identifications were based on both COI and $16 \mathrm{~S}$ genes. Our results show that a high proportion $(70 \%)$ of the so-called yak jerky sold in consumer markets of China is fake. Species mislabelling and adulteration can lead to economic and nutritional losses as well as food safety, health, and religious issues. Our result shows that three of the 30 samples were of meat from small wild mammals (e.g., shrew), which could be a host for infectious diseases and whose edibility is not known. The consumers' confidence regarding food quality and safety is decreasing currently, especially in China. Many severe cases of such adulteration, for example, of infant formula milk with melamine, adulteration with cooking oil, and artificial shark's fin, have been reported. Food traceability and correctly labeled composition are required to meet consumer requirements. The traditional method of morphology-based species identification fails in some of food adulterationassociated cases. DNA barcoding is widely used in food traceability analysis, e.g., for fish products (Filonzi et al., 2010), shark seafood (Barbuto et al., 2010), Amazonian fishes (Ardura et al., 2010), natural health products (Wallace et al., 2012), and olive oil (Kumar et al., 2011). Thus, this study reports another instance of the use of DNA barcoding in food traceability and shows that DNA barcoding is still a powerful tool to distinguish the species status of raw food materials, even for highly processed food like jerky.

Compliance and ethics The author(s) declare that they have no conflict of interest.

Acknowledgements We would like to thank Ke Jiang (Lasa), Liang Xie (Chengdu), and Kai Zhao (Qinghai) for collection of commercial yak jerky products. This work was supported by the National Natural Science Foundation of China (31090251), the Ministry of Science and Technology of China (2012FY110800), the Joint Grant of CAS Kunming Branch and Guizhou Academy of Sciences (2014-001), and the Animal Branch of the Germplasm Bank of Wild Species, Chinese Academy of Sciences (the Large Research Infrastructure Funding, Funding code: GBOWS to Weizhi Wang and Yaping Zhang).

Ardura, A., Linde, A.R., Moreira, J.C., and Garcia-Vazquez, E. (2010). DNA barcoding for conservation and management of Amazonian commercial fish. Biol Conserv 143, 1438-1443.

Barbuto, M., Galimberti, A., Ferri, E., Labra, M., Malandra, R., Galli, P., and Casiraghi, M. (2010). DNA barcoding reveals fraudulent substitutions in shark seafood products: the Italian case of "palombo" (Mustelus spp.). Food Res Int 43, 376-381.

Chen, S.Y., Liu, Y.P., and Yao, Y.G. (2010). Species authentication of commercial beef jerky based on PCR-RFLP analysis of the mitochondrial 12S rRNA gene. J Genet Genomics 37, 763-769.

Filonzi, L., Chiesa, S., Vaghi, M., and Marzano, F.N. (2010). Molecular barcoding reveals mislabelling of commercial fish products in Italy. Food Res Int 43, 1383-1388.

Galimberti, A., De Mattia, F., Losa, A., Bruni, I., Federici, S., Casiraghi, M., Martellos, S., and Labra, M. (2013). DNA barcoding as a new tool for food traceability. Food Res Int 50, 55-63.

Genearal Administration of Quality Supervision, I.a.Q., and Standardization Administration of China (2010). China National Standard of Yak dried beef. Beijing: Standards Press of China 9.

Hebert, P.D., Cywinska, A., Ball, S.L., and deWaard, J.R. (2003). Biological identifications through DNA barcodes. Proc Biol Sci 270, 313-321.

Kumar, S., Kahlon, T., and Chaudhary, S. (2011). A rapid screening for adulterants in olive oil using DNA barcodes. Food Chem 127, 1335-1341.

Qiu, Q., Zhang, G.J., Ma, T., Qian, W.B., Wang, J.Y., Ye, Z.Q., Cao, C.C., Hu, Q.J., Kim, J., Larkin, D.M., Auvil, L., Capitanu, B., Ma, J., Lewin, H.A., Qian, X.J., Lang, Y.S., Zhou, R., Wang, L.Z., Wang, K., Xia, J.Q., Liao, S.G., Pan, S.K., Lu, X., Hou, H.L., Wang, Y., Zang, X.T., Yin, Y., Ma, H., Zhang, J., Wang, Z.F., Zhang, Y.M., Zhang, D.W., Yonezawa, T., Hasegawa, M., Zhong, Y., Liu, W.B., Zhang, Y., Huang, Z.Y., Zhang, S.X., Long, R.J., Yang, H.M., Wang, J., Lenstra, J.A., Cooper, D.N., Wu, Y., Wang, J., Shi, P., Wang, J., and Liu, J.Q. (2012). The yak genome and adaptation to life at high altitude. Nat Genet 44, 946-949.

Ratnasingham, S., and Hebert, P.D. (2007). BOLD: The Barcode of Life Data System (http://www.barcodinglife.org). Mol Ecol Notes 7, 355-364.

Wallace, L.J., Boilard, S.M.A.L., Eagle, S.H.C., Spall, J.L., Shokralla, S., and Hajibabaei, M. (2012). DNA barcodes for everyday life: routine authentication of Natural Health Products. Food Res Int 49, 446-452.

Open Access This article is distributed under the terms of the Creative Commons Attribution License which permits any use, distribution, and reproduction in any medium, provided the original author(s) and source are credited.

\section{SUPPORTING INFORMATION}

Table S1 Sample information of commercial yak jerkies and their identification results.

The supporting information is available online at life.scichina.com and link.springer.com. The supporting materials are published as submitted, without typesetting or editing. The responsibility for scientific accuracy and content remains entirely with the authors. 